\title{
Editorial: Call for Papers Focused Collection of Physical Review Physics Education Research Qualitative Methods in PER: A Critical Examination
}

\author{
Guest Editors \\ Alexis V. Knaub, ${ }^{1}$ Katemari Rosa, ${ }^{2}$ and Ramón S. Barthelemy ${ }^{3}$
}

Physics Education Research (PER) uses various research methods classified under qualitative, quantitative, and mixed methods. These approaches help researchers understand physics education phenomena and advance our efforts to produce better PER. Over time, research questions and contexts have evolved, and so have our methods. We understand it has come the time for PER scholars to examine qualitative methods in our field critically. Therefore, we urge you to contribute to the Focused Collection on Qualitative Methods in PER.

Qualitative research in PER has been a foundational part of the field since its inception. This research approach is characterized by methods that draw upon non-numeric data; researchers often see qualitative research as a means of gaining a rich understanding of an area. Qualitative research can often be poignant for audiences, as such research can provide information in someone's own words connecting readers on a more personal level, promoting lasting insights.

Like any research methodology, qualitative methods inform researchers on possible research questions, types, and ways to collect data, and the lessons these data can provide. Gaining a rich understanding is of high interest for PER, from learning more about different physics learning spaces to getting detailed explanations on why and how one arrives at an answer. Qualitative methods in PER include but are not limited to the use of interviews, video transcripts, focus groups, portfolios, biographies, and other strategies that can yield a deep understanding of a specific physics education context or problem. All of these methods have assumptions, limitations, and other matters that impact what can be learned.

Although the PER community is cognoscente of qualitative research and frequently relies on qualitative methods, the field still lacks a broader use and discussion on many of its current and emergent practices. There is a wide range of qualitative research approaches and methods; they can vary in regards to theoretical perspectives, ways of data collection, coding, and analysis, as well as in the ways researchers make meaning of these data. Questions frequently arise about the validity and reliability of qualitative studies, with both researchers and audiences seeking to ensure qualitative studies are robust.

This Focused Collection on qualitative methods in physics education research aims to provide a space to examine and challenge current practices, learn how the qualitative landscape has changed over decades, and highlight the latest approaches in qualitative methods. We hope this publication will help the PER community to expand their tools for investigations. Manuscript proposals for this Focused Collection should not just use qualitative methods but should feature the qualitative methods themselves. As a critical examination, proposed manuscripts should include the strengths and weaknesses of the presented areas.

Some areas we envision this collection delving into include but are not limited to the following:

- Study design.-What types of data can answer what kinds of questions? How do the researchers engage with research participants at different stages of a project? How does the researcher impact data collection and interpretation? In what ways can qualitative researchers ensure their results are sound?

- Affordances and constraints of different methods. - What can and cannot be learned from different qualitative methods? Which methods may be more appropriate to answer particular research questions than others? What are the various limitations and advantages

\footnotetext{
${ }^{1}$ https://orcid.org/0000-0003-2028-3418

${ }^{2}$ https://orcid.org/0000-0002-0539-4104

${ }^{3}$ https://orcid.org/0000-0003-1225-7682
} 
of using one method over another in terms of, for example, the study's validity and reliability?

- Novel methods for collection and analysis. What new data sources are available today that were not available in the past? What are the latest methods and lenses being used to analyze data?

- Technology.-How have new technologies changed qualitative methodologies in terms of data collection and analysis? How and what new technologies are being used to develop qualitative studies? What are the ethics and other considerations?

- Data interpretation.- - How are the processes and techniques used for interpreting data in qualitative studies? How do researchers make sense of qualitative data?

We envision that authors may use qualitative data to highlight the utility of novel techniques for PER, use qualitative data to compare analysis techniques, conduct literature reviews to argue for or against particular qualitative methods, or engage in reflection that is embedded in data or literature. There may be other suitable forms. Ultimately, these manuscripts should provide insights on qualitative methods in or relevant to PER so researchers can make informed choices to produce high-quality qualitative research.

Submission Process.-Interested authors should submit 500-word proposals for full papers by 15 November 2021 to the guest editors, Alexis Knaub, Katemari Rosa, and Rámon Barthelemy, at prper.focusedcollection@gmail.com.

Proposals for empirical studies' manuscripts must include a brief discussion of the research methodology and data sources the work will expect to draw upon. Proposals for overview or review articles should provide a sampling of key references to be drawn upon in the article. Please include a sentence identifying the type of manuscript that is envisioned. Contact information and institutional affiliation of the lead author must be in the proposal. In consultation with the PRPER editor, the guest editors will review proposals and notify authors of their submission status by 15 January 2022. For proposals that are accepted, the authors will be asked to prepare manuscripts that will go through the standard PRPER review process.

Completed draft manuscripts will be due by 3 December 2022.-Given the short review cycle, we expect the submitted manuscripts to be complete works. Works in progress or incomplete articles will be returned to the author without review. We expect the Focused Collection to be published in summer 2023.

This Focused Collection will be supported by an advisory board comprised of Alan Alves-Brito (Federal University of Rio Grande do Sul, Brazil), Mildred Boveda (Penn State), Andréia Guerra (CEFET-RJ, Brazil), Danielle Harlow (University of California, Santa Barbara), Apriel Hodari (Eureka Scientific, Inc), and Silvania Sousa do Nascimento (Federal University of Minas Gerais, Brazil). Volunteers to review articles are welcome; please send a message to the guest editors at prper.focusedcollection@gmail.com.

Charles Henderson

Editor

Published 4 August 2021

DOI: 10.1103/PhysRevPhysEducRes.17.020001 